\title{
RECOVERY-BASED ERROR ESTIMATION AND ADAPTIVE SOLUTION OF ELLIPTIC VARIATIONAL INEQUALITIES OF THE SECOND KIND*
}

\author{
VIOREL BOSTAN ${ }^{\dagger}$ AND WEIMIN HAN ${ }^{\ddagger}$
}

\begin{abstract}
In this paper, we present and analyze gradient recovery type a posteriori error estimates for the finite element approximation of elliptic variational inequalities of the second kind. Both reliability and efficiency of the estimates are addressed. Some numerical results are reported, showing the effectiveness of the error estimates in adaptive solution of elliptic variational inequalities of the second kind.
\end{abstract}

\section{Introduction}

A posteriori error analysis has become an important tool for reliability assessment and efficiency improvement of the finite element method in solving both linear and nonlinear problems. An important class of a posteriori error estimates is based on local or global averaging of the gradient, e.g. in the form of Zienkiewicz-Zhu gradient recovery technique, $[19,20,21]$. It is known that in the case of structured grids and higher regularity solutions, such estimators are both efficient and reliable. Some work has been done for unstructured meshes as well, e.g. [16, 18]. A systematic study of various averaging techniques for a posteriori error estimation can be found in $[2,8]$.

The papers mentioned above are on a posteriori error estimation for solving elliptic boundary value problems of partial differential equations. For numerical solutions of variational inequalities, a few papers can be found for their a posteriori error analysis. E.g., residual type error estimators were obtained for an elliptic obstacle problem in [10] and for an elliptic variational inequality of the second kind in [5], and gradient recovery type error estimates for an elliptic obstacle problem have been shown recently in $[3,17]$.

In this paper, we derive and study a posteriori error estimates of gradient recovery type for finite element solutions of elliptic variational inequalities of the second kind. The paper is organized as follows. In Section 2 we introduce the model problem. Section 3 contains the finite element method setting. In Section 4 we derive a posteriori error estimates based on both global and local averaging techniques. Section 5 is devoted to an analysis of the efficiency of the estimators. In Section 6 we report some numerical results. We restrict ourselves in this paper to linear elements since they are popularly used in solving variational inequalities due to a lack of higher solution regularity. Also, our discussion focuses on one particular gradient recovery technique. The discussion and results presented here can be extended to other elements and other averaging techniques as studied in $[2,8]$.

We now list some notations used in the paper. Let $\Omega$ be a bounded domain in $\mathbb{R}^{d}, d \geq 1$, with Lipschitz boundary $\Gamma=\partial \Omega$. Let $\Gamma_{D}$ be a closed subset of $\Gamma$ with meas $\left(\Gamma_{D}\right)>0$ and $\Gamma_{C}$ be the remaining part. For any open subset $\omega$ of $\Omega$ with Lipschitz boundary $\partial \omega$, we denote by $H^{m}(\omega), L^{2}(\omega)$ and $L^{2}(\partial \omega)$ the usual Sobolev

*Received: December 4, 2003; accepted (in revised version): January 15, 2004. Communicated by Shi Jin.

This work was supported by NSF under grant DMS-0106781.

$\dagger$ Department of Mathematics, University of Iowa, Iowa City, IA 52242, U.S.A, (vbostan@math.uiowa.edu).

${ }^{\ddagger}$ Department of Mathematics, University of Iowa Iowa City, IA 52242, U.S.A, (whan@math.uiowa.edu). 
and Lebesgue spaces with the standard norms $\|\cdot\|_{m ; \omega}:=\|\cdot\|_{H^{m}(\omega)},\|\cdot\|_{0 ; \omega}:=\|\cdot\|_{L^{2}(\omega)}$ and $\|\cdot\|_{0 ; \partial \omega}:=\|\cdot\|_{L^{2}(\partial \omega)}$. Also, we will make use of the standard seminorm $|\cdot|_{m, \omega}:=$ $\left\|D^{m} \cdot\right\|_{0 ; \omega}$ on $H^{m}(\omega)$. Throughout this paper we will use the same notation $v$ to denote both $v \in H^{1}(\Omega)$ and its trace $\gamma v \in L^{2}(\Gamma)$ on the boundary. We reserve the symbol $\gamma$ to denote sides of the finite elements.

\section{Model problem}

The discussion of the a posteriori error estimates in this paper is focused on a model elliptic variational inequality of the second kind. Such a variational inequality is featured by the presence of non-differentiable terms in the formulation. The techniques and results presented in this paper can be extended to general elliptic variational inequalities of the second kind in a straightforward fashion.

Let there be given $f \in L^{2}(\Omega)$ and a constant $g>0$. Over the space $V=\{v \in$ $H^{1}(\Omega) \mid v=0$ on $\left.\Gamma_{D}\right\}$, we define

$$
\begin{aligned}
a(u, v) & =\int_{\Omega}(\nabla u \cdot \nabla v+u v) d x, \\
l(v) & =\int_{\Omega} f v d x \\
j(v) & =\int_{\Gamma_{C}} g|v| d s .
\end{aligned}
$$

The model problem is the following elliptic variational inequality of the second kind: Find $u \in V$ such that

$$
a(u, v-u)+j(v)-j(u) \geq l(v-u) \quad \forall v \in V .
$$

This model is a so-called simplified friction problem (cf. [13]) as it can be viewed as a simplified version of a frictional contact problem in linearized elasticity. Existence and uniqueness of a solution for the problem (2.1) follow from a classical result on elliptic variational inequalities (see e.g. [13] or [14]).

In the analysis of a posteriori error estimators later, we will need the following characterization of the solution $u$ of $(2.1)$ :

There exists a unique $\lambda \in L^{\infty}\left(\Gamma_{C}\right)$ such that

$$
\begin{aligned}
& a(u, v)+\int_{\Gamma_{C}} g \lambda v d s=l(v) \quad \forall v \in V, \\
& |\lambda| \leq 1, \quad \lambda u=|u| \quad \text { on } \Gamma_{C} .
\end{aligned}
$$

A proof of this characterization in the case $\Gamma_{C}=\Gamma$ can be found in [13]; the proof can be easily extended to the general case, cf. [6]. It follows from the above characterization that the solution $u$ of (2.1) is the weak solution of the boundary value problem

$$
\begin{aligned}
& -\Delta u+u=f \quad \text { in } \Omega \text {, } \\
& \frac{\partial u}{\partial n}+g \lambda=0 \quad \text { on } \Gamma_{C}, \\
& u=0 \quad \text { on } \Gamma_{D} .
\end{aligned}
$$

Here, $\partial / \partial n$ denotes the outward normal derivative. 


\section{Finite element approximation}

For simplicity, we suppose that $\Omega$ has a polyhedral boundary $\Gamma$. In order to define the finite element method for (2.1) we introduce a family of regular partitions $\mathcal{P}_{h}$ of $\bar{\Omega}$ consisting of triangles $(d=2)$ or tetrahedrons $(d=3)$. The partitions $\mathcal{P}_{h}$ are compatible with the decomposition of $\partial \Omega$ into $\Gamma_{D}$ and $\Gamma_{C}$. In other words, if an element side lies on the boundary, then it belongs to one of the sets $\Gamma_{D}$ or $\overline{\Gamma_{C}}$. For every element $K \in \mathcal{P}_{h}$, let $h_{K}$ be the diameter of $K$ and $\rho_{K}$ be the diameter of the largest ball inscribed in $K$. For a side $\gamma$ of the element $K$, we denote by $h_{\gamma}$ the diameter of $\gamma$. We shall assume that the family of partitions $\mathcal{P}_{h}, h>0$, satisfies the shape regularity assumption, i.e. the ratio $h_{K} / \rho_{K}$ is uniformly bounded over the whole family by a constant $C$. Note that the shape regularity assumption does not require that the elements be of comparable size and thus locally refined meshes are allowed. Define the finite element spaces

$$
S_{h}=\left\{v_{h} \in C(\bar{\Omega})\left|v_{h}\right|_{K} \text { is affine } \forall K \in \mathcal{P}_{h}\right\}
$$

and

$$
V_{h}=\left\{v_{h} \in S_{h} \mid v_{h}=0 \text { on } \Gamma_{D}\right\} .
$$

We will use $\mathcal{E}_{h}$ for the set of the element sides, $\mathcal{E}_{h, C}$ for the subset of the element sides lying on the $\overline{\Gamma_{C}}$ part of the boundary, and $\mathcal{E}_{h, 0}$ is the subset of the element sides that do not lie on $\Gamma$. Let $\mathcal{N}_{h}$ be the set of all vertices in $\mathcal{P}_{h}$ and $\mathcal{N}_{h, 0} \subset \mathcal{N}_{h}$ the set of free vertices, i.e. those vertices that do not lie on $\Gamma_{D}$. For a given element $K \in \mathcal{P}_{h}$, $\mathcal{N}(K)$ and $\mathcal{E}(K)$ denote the sets of the vertices of $K$ and sides of $K$, respectively. The patch $\widetilde{K}$ associated with any element $K$ from a partition $\mathcal{P}_{h}$ consists of all elements sharing at least one vertex with $K$, i.e. $\widetilde{K}=\bigcup\left\{K^{\prime} \in \mathcal{P}_{h}: \overline{K^{\prime}} \cap \bar{K} \neq \emptyset\right\}$. Similarly, for any side $\gamma \in \mathcal{E}_{h}$, the patch $\widetilde{\gamma}$ consists of the elements sharing $\gamma$ as a common side. Note that in the case when the side $\gamma$ lies on the boundary $\Gamma$, the patch $\widetilde{\gamma}$ consists of only one element. For a given element $K \in \mathcal{P}_{h}$ or a given side $\gamma \in \mathcal{E}_{h}, n_{K}$ or $n_{\gamma}$ denotes the unit normal vector to the sides of $K$ or to the side $\gamma$, respectively. In what follows, for any piecewise continuous function $\varphi$ and any interior side $\gamma \in \mathcal{E}_{h, 0}$, $[\varphi]_{\gamma}$ denotes the jump of $\varphi$ across $\gamma$ in the direction $n_{\gamma}$, i.e.

$$
[\varphi]_{\gamma}(x)=\lim _{t \rightarrow 0+}\left(\varphi\left(x+t n_{\gamma}\right)-\varphi\left(x-t n_{\gamma}\right)\right), \quad x \in \gamma .
$$

The discretization of variational inequality (2.1) is defined by the Galerkin finite element method: Find $u_{h} \in V_{h}$ such that

$$
a\left(u_{h}, v_{h}-u_{h}\right)+j\left(v_{h}\right)-j\left(u_{h}\right) \geq l\left(v_{h}-u_{h}\right) \quad \forall v_{h} \in V_{h} .
$$

We need the following characterization of the finite element solution (cf. [6]), similar to that of the solution of the continuous problem.

THEOREM 3.1. The unique solution $u_{h} \in V_{h}$ of the discretized problem (3.1) is characterized by the existence of $\lambda_{h} \in L^{\infty}\left(\Gamma_{C}\right)$ such that

$$
\begin{aligned}
& a\left(u_{h}, v_{h}\right)+\int_{\Gamma_{C}} g \lambda_{h} v_{h} d s=l\left(v_{h}\right) \quad \forall v_{h} \in V_{h}, \\
& \left|\lambda_{h}\right| \leq 1, \quad \lambda_{h} u_{h}=\left|u_{h}\right| \quad \text { a.e. on } \Gamma_{C} .
\end{aligned}
$$


In the derivation of the a posteriori error estimate we use the so-called weighted Clément-type interpolation operator. There are several variants of such operators (see e.g. $[2,7,8,9,17])$, which are all versions of the interpolation operator introduced by Clément [11]. The main difference among these interpolants lies in the way the interpolation is performed near the boundary. In this paper we will follow the approach used in [8].

Given a vertex $A \in \mathcal{N}_{h}$, let $\varphi_{A} \in S_{h}$ be the nodal basis function associated with $A$. For each fixed vertex $A \in \Gamma_{D}$, choose $\xi(A) \in \mathcal{N}_{h, 0}$ to be a vertex of an element containing $A$. Let $\xi(A)=A$ if $A \in \mathcal{N}_{h, 0}$. For each vertex $A \in \mathcal{N}_{h, 0}$ define the class $I(A)=\left\{\tilde{A} \in \mathcal{N}_{h} \mid \xi(\tilde{A})=A\right\}$. In this way, the set of all vertices $\mathcal{N}_{h}$ is partitioned into $\operatorname{card}\left(\mathcal{N}_{h, 0}\right)$ classes of equivalence. For each $A \in \mathcal{N}_{h, 0}$ set

$$
\psi_{A}=\sum_{\xi \in I(A)} \varphi_{\xi}
$$

and notice that $\left\{\psi_{A} \mid A \in \mathcal{N}_{h, 0}\right\}$ is a partition of unity. Let $\widetilde{K}_{A}=\operatorname{supp}\left(\psi_{A}\right)$ and $h_{A}=\operatorname{diam}\left(\widetilde{K}_{A}\right)$. It is evident that $\widetilde{K}_{A}$ is connected and that $\psi_{A} \neq \varphi_{A}$ implies that $\Gamma_{D} \cap \widetilde{K}_{A}$ has a positive surface measure. For a given $v \in L^{1}(\Omega)$, let

$$
v_{A}=\frac{\int_{\widetilde{K}_{A}} \psi_{A} v d x}{\int_{\widetilde{K}_{A}} \varphi_{A} d x}, \quad A \in \mathcal{N}_{h, 0} .
$$

Then define the interpolation operator $\Pi_{h}: V \rightarrow V_{h}$ as follows:

$$
\Pi_{h} v=\sum_{A \in \mathcal{N}_{h, 0}} v_{A} \varphi_{A} .
$$

The next result summarizes some basic estimates for $\Pi_{h}$. Its proof can be found in $[8]$.

THEOREM 3.2. There exists an $h$-independent constant $C>0$ such that for all $v \in V$ and $f \in L^{2}(\Omega)$ there holds

$$
\begin{aligned}
\left|v-\Pi_{h} v\right|_{1 ; \Omega}^{2} & \leq C|v|_{1 ; \Omega}^{2} \\
\int_{\Omega} f\left(v-\Pi_{h} v\right) d x & \leq C|v|_{1 ; \Omega}\left(\sum_{A \in \mathcal{N}_{h}, 0} h_{A}^{2} \min _{f_{A} \in \mathbb{R}}\left\|f-f_{A}\right\|_{0 ; \widetilde{K}_{A}}^{2}\right)^{1 / 2} \\
\sum_{K \in \mathcal{P}_{h}}\left\|h_{K}^{-1}\left(v-\Pi_{h} v\right)\right\|_{0 ; K}^{2} & \leq C|v|_{1 ; \Omega}^{2} \\
\sum_{\gamma \in \mathcal{E}_{h}}\left\|h_{\gamma}^{-1 / 2}\left(v-\Pi_{h} v\right)\right\|_{0 ; \gamma}^{2} & \leq C|v|_{1 ; \Omega}^{2} .
\end{aligned}
$$

\section{Gradient recovery-based a posteriori error estimate}

The a posteriori error estimate presented in this paper is derived from the following theorem, which is given in [6], in a slightly different problem setting. Its proof makes use of the duality theory in convex analysis.

THEOREM 4.1. Let $u \in V$ be the unique solution of (2.1), and $w \in V$ an approximation of $u$. Then for any $\boldsymbol{r}^{*}=\left(\boldsymbol{r}_{1}^{*}, r_{2}^{*}, r_{3}^{*}\right) \in\left(L^{2}(\Omega)\right)^{d} \times L^{2}(\Omega) \times L^{2}\left(\Gamma_{C}\right),\left|r_{3}^{*}\right| \leq g$ a.e. 
on $\Gamma_{C}$, the following error bound is valid:

$$
\begin{aligned}
\frac{1}{2} a(u-w, u-w) \leq & \int_{\Omega}\left(\left|\nabla w+\boldsymbol{r}_{1}^{*}\right|^{2}+\left|w+r_{2}^{*}\right|^{2}\right) d x \\
& +\mathcal{R}\left(\boldsymbol{r}^{*}\right)^{2}+\int_{\Gamma_{C}}\left(g|w|+r_{3}^{*} w\right) d s,
\end{aligned}
$$

where the residual $\mathcal{R}\left(\boldsymbol{r}^{*}\right)$ is given by

$$
\mathcal{R}\left(\boldsymbol{r}^{*}\right)=\sup _{v \in V} \frac{1}{\|v\|_{V}}\left\{\int_{\Omega}\left(\boldsymbol{r}_{1}^{*} \cdot \nabla v+\left(r_{2}^{*}+f\right) v\right) d x+\int_{\Gamma_{C}} r_{3}^{*} v d s\right\} .
$$

In Theorem 4.1, $w$ can be any approximation of the solution $u$, though we will only use the Galerkin finite element solution $u_{h}$ in the following. Theorem 4.1 provides a general framework for deriving various a posteriori error estimates with different choices of the auxiliary variable $\boldsymbol{r}^{*}$. For example, residual-type error estimates were derived in [6] by choosing $\boldsymbol{r}^{*}=-\left(\nabla u_{h}, u_{h}, g \lambda_{h}\right)$, where $u_{h}$ is the finite element solution and $\lambda_{h}$ is provided by Theorem 3.1. In this paper we consider a different choice for auxiliary variable $\boldsymbol{r}^{*}$, which will lead to a gradient recovery type a posteriori error estimates.

To formulate the error estimator we need a gradient recovery operator. There are many types of gradient recovery operators. In order to have a "good" approximation of the true gradient $\nabla u$, a set of conditions to be satisfied by the recovery operator were identified in [1]. These conditions lead to a more precise characterization of the form of the gradient recovery operator, summarized in Lemma 4.5 in [1]. In particular, the recovered gradient at a vertex $A$ is a linear combination of the values of $\nabla u_{h}$ in a patch surrounding $A$.

We define the gradient recovery operator $G_{h}: V_{h} \rightarrow\left(V_{h}\right)^{d}$ as follows

$$
G_{h} v_{h}=\sum_{A \in \mathcal{N}_{h}} G_{h} v_{h}(A) \varphi_{A}, \quad G_{h} v_{h}(A)=\frac{1}{\left|\widetilde{K}_{A}\right|} \int_{\widetilde{K}_{A}} \nabla v_{h} d x
$$

Since linear elements are used,

$$
G_{h} v_{h}(A)=\sum_{i=1}^{N_{A}} \alpha_{A}^{i}\left(\nabla v_{h}\right)_{K_{A}^{i}}
$$

where $\left(\nabla v_{h}\right)_{K_{A}^{i}}$ denotes the vector value of the gradient $\nabla v_{h}$ on the element $K_{A}^{i}$, $\widetilde{K}_{A}=\bigcup_{i=1}^{N_{A}} K_{A}^{i}, \alpha_{A}^{i}=\left|K_{A}^{i}\right| /\left|\widetilde{K}_{A}\right|, i=1, \ldots, N_{A}$.

With the finite element solution $u_{h}$, the error estimator based on the gradient recovery can be defined as follows:

$\eta_{G}^{2}=\sum_{K \in \mathcal{P}_{h}} \eta_{K, G}^{2}, \quad \eta_{K, G}^{2}=\left\|\nabla u_{h}-G_{h} u_{h}\right\|_{0 ; K}^{2}+\sum_{\gamma \in \mathcal{E}(K) \cap \mathcal{E}_{h, C}} h_{\gamma}\left\|G_{h} u_{h} \cdot n_{\gamma}+g \lambda_{h}\right\|_{0 ; \gamma}^{2}$.

THEOREM 4.2. Let $u$ and $u_{h}$ be the unique solutions of (2.1) and (3.1), respectively. Then the error $u-u_{h}$ satisfies the a posteriori estimate

$$
\left\|u-u_{h}\right\|_{1 ; \Omega}^{2} \leq C \eta_{G}^{2}+C \sum_{A \in \mathcal{N}_{h}, 0}\left(h_{A}^{4}\left\|\nabla u_{h}\right\|_{0 ; \widetilde{K}_{A}}^{2}+h_{A}^{2} \min _{f_{A} \in \mathbb{R}}\left\|f-f_{A}\right\|_{0 ; \widetilde{K}_{A}}^{2}\right) .
$$


Proof. Let $u_{h}$ be the finite element solution, $\lambda_{h} \in L^{\infty}\left(\Gamma_{C}\right)$ be provided by Theorem 3.1 and $G_{h} u_{h}$ be the recovered gradient defined in (4.3). Apply Theorem 4.1 with $w=u_{h}$ and $\boldsymbol{r}^{*}=-\left(G_{h} u_{h}, u_{h}, g \lambda_{h}\right)$ to obtain

$$
\frac{1}{2} a\left(u-u_{h}, u-u_{h}\right) \leq \int_{\Omega}\left|\nabla u_{h}-G_{h} u_{h}\right|^{2} d x+\mathcal{R}^{2},
$$

where residual $\mathcal{R}$ is given by

$$
\mathcal{R}=\sup _{v \in V} \frac{1}{\|v\|_{V}}\left\{\int_{\Omega}\left[G_{h} u_{h} \cdot \nabla v+\left(u_{h}-f\right) v\right] d x+\int_{\Gamma_{C}} g \lambda_{h} v d s\right\} .
$$

Let $\Pi_{h}$ be the interpolation operator defined by (3.4). Use characterization (3.2) with $v_{h}=\Pi_{h} v$ to get

$$
\int_{\Omega}\left[\nabla u_{h} \cdot \nabla \Pi_{h} v+\left(u_{h}-f\right) \Pi_{h} v\right] d x+\int_{\Gamma_{C}} g \lambda_{h} \Pi_{h} v d s=0 .
$$

Therefore, we can rewrite (4.8) as

$$
\begin{aligned}
\mathcal{R}=\sup _{v \in V} & \frac{1}{\|v\|_{V}}\left\{\int_{\Omega}\left[G_{h} u_{h} \cdot \nabla\left(v-\Pi_{h} v\right)+\left(u_{h}-f\right)\left(v-\Pi_{h} v\right)\right] d x\right. \\
& \left.+\int_{\Gamma_{C}} g \lambda_{h}\left(v-\Pi_{h} v\right) d s+\int_{\Omega}\left(G_{h} u_{h}-\nabla u_{h}\right) \cdot \nabla \Pi_{h} v d x\right\} .
\end{aligned}
$$

By estimate (3.5), we have $\left\|\nabla \Pi_{h} v\right\|_{0 ; \Omega} \leq C\|v\|_{V}$ and so

$$
\sup _{v \in V} \frac{1}{\|v\|_{V}} \int_{\Omega}\left(G_{h} u_{h}-\nabla u_{h}\right) \cdot \nabla \Pi_{h} v d x \leq C\left\|\nabla u_{h}-G_{h} u_{h}\right\|_{0 ; \Omega}
$$

Thus,

$$
\mathcal{R} \leq C\left\|\nabla u_{h}-G_{h} u_{h}\right\|_{0 ; \Omega}+\mathcal{R}_{1}
$$

where residual $\mathcal{R}_{1}$ is given by

$\mathcal{R}_{1}=\sup _{v \in V} \frac{1}{\|v\|_{V}}\left\{\int_{\Omega}\left[G_{h} u_{h} \cdot \nabla\left(v-\Pi_{h} v\right)+\left(u_{h}-f\right)\left(v-\Pi_{h} v\right)\right] d x+\int_{\Gamma_{C}} g \lambda_{h}\left(v-\Pi_{h} v\right) d s\right\}$.

Integrate by parts over each element $K \in \mathcal{P}_{h}$ to get

$$
\begin{aligned}
\mathcal{R}_{1}=\sup _{v \in V} \frac{1}{\|v\|_{V}} \sum_{K \in \mathcal{P}_{h}}\left\{\int_{K}\left(-\operatorname{div}\left(G_{h} u_{h}\right)+u_{h}-f\right)\left(v-\Pi_{h} v\right) d x\right. \\
\left.+\sum_{\gamma \in \mathcal{E}(K)} \int_{\gamma} G_{h} u_{h} \cdot n_{\gamma}\left(v-\Pi_{h} v\right) d s+\sum_{\gamma \in \mathcal{E}(K) \cap \mathcal{E}_{h, C}} \int_{\gamma} g \lambda_{h}\left(v-\Pi_{h} v\right) d s\right\} .
\end{aligned}
$$

Since $G_{h} u_{h}$ is continuous, the integrals on interior sides $\gamma \in \mathcal{E}_{h, 0}$ in (4.10) cancel each other. Rewrite

$$
-\operatorname{div}\left(G_{h} u_{h}\right)+u_{h}-f=\operatorname{div}\left(\nabla u_{h}-G_{h} u_{h}\right)+\left(-\Delta u_{h}+u_{h}-f\right)
$$


and rearrange the terms in (4.10) to obtain

$$
\begin{aligned}
\mathcal{R}_{1}=\sup _{v \in V} \frac{1}{\|v\|_{V}} & \left\{\sum_{K \in \mathcal{P}_{h}} \int_{K} \operatorname{div}\left(\nabla u_{h}-G_{h} u_{h}\right)\left(v-\Pi_{h} v\right) d x\right. \\
& +\sum_{K \in \mathcal{P}_{h}} \int_{K} r_{K}\left(v-\Pi_{h} v\right) d x \\
& \left.+\sum_{\gamma \in \mathcal{E}_{h, C}} \int_{\gamma}\left(G_{h} u_{h} \cdot n_{\gamma}+g \lambda_{h}\right)\left(v-\Pi_{h} v\right) d s\right\} \\
= & \sup _{v \in V} \frac{1}{\|v\|_{V}}\{I+I I+I I I\},
\end{aligned}
$$

where $r_{K}=-\Delta u_{h}+u_{h}-f=u_{h}-f$ denotes the interior residual on element $K \in \mathcal{P}_{h}$. We will use $r$ to denote the piecewise interior residual, i.e., $\left.r\right|_{K}=r_{K}$ for $K \in \mathcal{P}_{h}$. To estimate the first summand on the right side of (4.11), we use an elementwise inverse inequality of the form

$$
\left\|\operatorname{div}\left(\nabla u_{h}-G_{h} u_{h}\right)\right\|_{0 ; K} \leq C h_{K}^{-1}\left\|\nabla u_{h}-G_{h} u_{h}\right\|_{0 ; K} .
$$

Apply the Cauchy-Schwarz inequality, the inverse inequality (4.12) and the estimate (3.7) to get

$$
\begin{aligned}
I & \leq C \sum_{K \in \mathcal{P}_{h}}\left\|\operatorname{div}\left(\nabla u_{h}-G_{h} u_{h}\right)\right\|_{0 ; K}\left\|v-\Pi_{h} v\right\|_{0 ; K} \\
& \leq C \sum_{K \in \mathcal{P}_{h}}\left\|\nabla u_{h}-G_{h} u_{h}\right\|_{0 ; K}\left\|h_{K}^{-1}\left(v-\Pi_{h} v\right)\right\|_{0 ; K} \\
& \leq C\left(\sum_{K \in \mathcal{P}_{h}}\left\|\nabla u_{h}-G_{h} u_{h}\right\|_{0 ; K}^{2}\right)^{1 / 2}\left(\sum_{K \in \mathcal{P}_{h}}\left\|h_{K}^{-1}\left(v-\Pi_{h} v\right)\right\|_{0 ; K}^{2}\right)^{1 / 2} \\
& \leq C|v|_{1 ; \Omega}\left(\sum_{K \in \mathcal{P}_{h}}\left\|\nabla u_{h}-G_{h} u_{h}\right\|_{0 ; K}^{2}\right)^{1 / 2} .
\end{aligned}
$$

For the second summand, we apply the estimate (3.6) to obtain

$$
I I \leq C|v|_{1 ; \Omega}\left(\sum_{A \in \mathcal{N}_{h, 0}} h_{A}^{2} \min _{r_{A} \in \mathbb{R}}\left\|r-r_{A}\right\|_{0 ; \widetilde{K}_{A}}^{2}\right)^{1 / 2} .
$$

Now

$$
\begin{aligned}
\sum_{A \in \mathcal{N}_{h, 0}} h_{A}^{2} \min _{r_{A} \in \mathbb{R}}\left\|r-r_{A}\right\|_{0 ; \widetilde{K}_{A}}^{2} \leq & 2 \sum_{A \in \mathcal{N}_{h, 0}} h_{A}^{2}\left\|u_{h}-\overline{u_{h}}\right\|_{0 ; \widetilde{K}_{A}}^{2} \\
& +2 \sum_{A \in \mathcal{N}_{h, 0}} h_{A}^{2} \min _{f_{A} \in \mathbb{R}}\left\|f-f_{A}\right\|_{0 ; \widetilde{K}_{A}}^{2},
\end{aligned}
$$

where $\overline{u_{h}}$ denotes the integral mean of $u_{h}$ over $\widetilde{K}_{A}$. Use the Poincare inequality and 
an inverse inequality of the form (4.12) to get

$$
\begin{aligned}
\sum_{A \in \mathcal{N}_{h, 0}} h_{A}^{2} \min _{r_{A} \in \mathbb{R}}\left\|r-r_{A}\right\|_{0 ; \widetilde{K}_{A}}^{2} \leq & C \sum_{A \in \mathcal{N}_{h, 0}} h_{A}^{4}\left\|\nabla u_{h}\right\|_{0 ; \widetilde{K}_{A}}^{2} \\
& +2 \sum_{A \in \mathcal{N}_{h, 0}} h_{A}^{2} \min _{f_{A} \in \mathbb{R}}\left\|f-f_{A}\right\|_{0 ; \widetilde{K}_{A}}^{2} .
\end{aligned}
$$

Therefore,

$$
I I \leq C|v|_{1 ; \Omega} \sum_{A \in \mathcal{N}_{h, 0}}\left(h_{A}^{4}\left\|\nabla u_{h}\right\|_{0 ; \widetilde{K}_{A}}^{2}+h_{A}^{2} \min _{f_{A} \in \mathbb{R}}\left\|f-f_{A}\right\|_{0 ; \tilde{K}_{A}}^{2}\right)
$$

Finally, with the aid of the Cauchy-Schwarz inequality and the estimate (3.8), the third summand on the right side of (4.11) can be bounded by

$$
I I I \leq C|v|_{1 ; \Omega}\left(\sum_{\gamma \in \mathcal{E}_{h, C}} h_{\gamma}\left\|G_{h} u_{h} \cdot n_{\gamma}+g \lambda_{h}\right\|_{0 ; \gamma}^{2}\right)^{1 / 2} .
$$

Inserting (4.13), (4.14) and (4.15) into (4.11), and using the Cauchy-Schwarz inequality and (4.9), we deduce that

$$
\begin{aligned}
\mathcal{R} \leq C & \left\{\sum_{K \in \mathcal{P}_{h}}\left\|\nabla u_{h}-G_{h} u_{h}\right\|_{0 ; K}^{2}+\sum_{\gamma \in \mathcal{E}_{h, C}} h_{\gamma}\left\|G_{h} u_{h} \cdot n_{\gamma}+g \lambda_{h}\right\|_{0 ; \gamma}^{2}\right. \\
& \left.+\sum_{A \in \mathcal{N}_{h, 0}}\left(h_{A}^{4}\left\|\nabla u_{h}\right\|_{0 ; \tilde{K}_{A}}^{2}+h_{A}^{2} \min _{f_{A} \in \mathbb{R}}\left\|f-f_{A}\right\|_{0 ; \tilde{K}_{A}}^{2}\right)\right\}^{1 / 2} .
\end{aligned}
$$

Split the first term on the right side of estimate (4.7) into local contributions from each $K \in \mathcal{P}_{h}$ and insert (4.16) to conclude the proof.

We can write (4.6) as

$$
\left\|u-u_{h}\right\|_{1 ; \Omega} \leq C \eta_{G}+R_{h}
$$

where

$$
R_{h}=C\left[\sum_{A \in \mathcal{N}_{h, 0}} h_{A}^{4}\left\|\nabla u_{h}\right\|_{0 ; \widetilde{K}_{A}}^{2}\right]^{1 / 2}+C\left[\sum_{A \in \mathcal{N}_{h, 0}} h_{A}^{2} \min _{f_{A} \in \mathbb{R}}\left\|f-f_{A}\right\|_{0 ; \widetilde{K}_{A}}^{2}\right]^{1 / 2} .
$$

We observe that usually the term $R_{h}$ is of higher order compared to $\left\|u-u_{h}\right\|_{1 ; \Omega}$ which is of order $O(h)$ in the nondegenerate situations. This observation is argued as follows: First, it is easy to show from the definition of the finite element solutions that there is a constant $C$ such that $\left\|u_{h}\right\|_{1 ; \Omega} \leq C$ for any $h$. So the term

$$
\left[\sum_{A \in \mathcal{N}_{h, 0}} h_{A}^{4}\left\|\nabla u_{h}\right\|_{0 ; \widetilde{K}_{A}}^{2}\right]^{1 / 2}
$$


is bounded by $O\left(h^{2}\right)$. Next, for $f \in L^{2}(\Omega)$,

$$
\left(\sum_{A \in \mathcal{N}_{h, 0}} h_{A}^{2} \min _{f_{A} \in \mathbb{R}}\left\|f-f_{A}\right\|_{0 ; \widetilde{K}_{A}}^{2}\right)^{1 / 2}=o(h) ;
$$

and if $f \in H^{1}(\Omega)$, then

$$
\left(\sum_{A \in \mathcal{N}_{h, 0}} h_{A}^{2} \min _{f_{A} \in \mathbb{R}}\left\|f-f_{A}\right\|_{0 ; \widetilde{K}_{A}}^{2}\right)^{1 / 2}=O\left(h^{2}\right) .
$$

Thus, (4.17) illustrates the reliability of the error estimator $\eta_{G}$.

\section{Efficiency of the estimator}

In what follows, we investigate the efficiency of the error estimator $\eta_{G}$. For this purpose, we will prove that for each element $K \in \mathcal{P}_{h}, \eta_{K, G}$ is dominated by the residual-type estimator $\eta_{K, R}$ derived in [6]:

$$
\eta_{K, R}^{2}=h_{K}^{2}\left\|r_{K}\right\|_{0 ; K}^{2}+\sum_{\gamma \in \mathcal{E}(K)} h_{\gamma}\left\|R_{\gamma}\right\|_{0 ; \gamma}^{2}
$$

with the interior residual $r_{K}=-\Delta u_{h}+u_{h}-f=u_{h}-f$ in $K \in \mathcal{P}_{h}$ and side residual

$$
R_{\gamma}= \begin{cases}{\left[\frac{\partial u_{h}}{\partial n}\right]_{\gamma}} & \text { if } \gamma \in \mathcal{E}_{h, 0} \\ \frac{\partial u_{h}}{\partial n}+g \lambda_{h} & \text { if } \gamma \in \mathcal{E}_{h, C}\end{cases}
$$

For the error estimator $\eta_{R}^{2}=\sum_{K \in \mathcal{P}_{h}} \eta_{K, R}^{2}$, we have proved in [6] the following estimate

$$
\begin{aligned}
\eta_{R}^{2} \leq C & \left(\left\|u-u_{h}\right\|_{1 ; \Omega}^{2}+\sum_{\gamma \in \mathcal{E}_{h, C}} h_{\gamma}\left\|\lambda-\lambda_{h}\right\|_{0 ; \gamma}^{2}\right. \\
& \left.+\sum_{K \in \mathcal{P}_{h}} h_{K}^{2}\left\|r_{K}-\overline{r_{K}}\right\|_{0 ; K}^{2}+\sum_{\gamma \in \mathcal{E}_{h, C}} h_{\gamma}\left\|R_{\gamma}-\overline{R_{\gamma}}\right\|_{0 ; \gamma}^{2}\right)
\end{aligned}
$$

with discontinuous piecewise polynomial approximations $\overline{r_{K}}, \overline{R_{\gamma}}$ of $r_{K}, R_{\gamma}$.

Lemma 5.1. Let $\eta_{K, G}$ be defined by (4.5). Then the following bound holds

$$
\eta_{K, G}^{2} \leq C\left(\sum_{\gamma \in \mathcal{E}(K) \cap \mathcal{E}_{h, C}} h_{\gamma}\left\|R_{\gamma}\right\|_{0 ; \gamma}^{2}+\sum_{\gamma^{\prime} \in \mathcal{E}_{\widetilde{K}}} h_{\gamma^{\prime}}\left\|R_{\gamma^{\prime}}\right\|_{0 ; \gamma^{\prime}}^{2}\right),
$$

where $\mathcal{E}_{\widetilde{K}}$ denotes the set of inner edges of the patch $\widetilde{K}$ corresponding to the element $K$.

Proof. It follows from the definition of $G_{h}$ that on each element $K$,

$$
\begin{aligned}
\left|\nabla u_{h}-G_{h} u_{h}\right|^{2} & =\left|\sum_{A \in \mathcal{N}(K)} \varphi_{A}\left(\sum_{i=1}^{N_{A}} \alpha_{A}^{i}\left(\left(\nabla u_{h}\right)_{K}-\left(\nabla u_{h}\right)_{K_{A}^{i}}\right)\right)\right|^{2} \\
& \leq C \sum_{K^{\prime} \subset \widetilde{K}}\left|\left(\nabla u_{h}\right)_{K}-\left(\nabla u_{h}\right)_{K^{\prime}}\right|^{2} .
\end{aligned}
$$


For any $K^{\prime} \subset \widetilde{K}$ there is a sequence of inner edges $\gamma_{1}, \ldots, \gamma_{m}$, such that $\widetilde{\gamma}_{j} \cap \widetilde{\gamma}_{j+1} \neq \emptyset$ and $K \subset \widetilde{\gamma_{1}}, K^{\prime} \subset \widetilde{\gamma_{m}}$. Hence,

$$
\left|\left(\nabla u_{h}\right)_{K}-\left(\nabla u_{h}\right)_{K^{\prime}}\right| \leq\left|\sum_{j=1}^{m}\left[\nabla u_{h}\right]_{\gamma_{j}}\right| \leq \sum_{j=1}^{m}\left|\left[\nabla u_{h}\right]_{\gamma_{j}}\right| \leq \sum_{\gamma \in \mathcal{E}_{\widetilde{K}}}\left|\left[\nabla u_{h}\right]_{\gamma}\right| .
$$

Since $u_{h}$ is continuous in $\bar{\Omega},\left[\partial u_{h} / \partial t\right]_{\gamma}=0$ for all $\gamma \in \mathcal{E}_{h, 0}$, where $\partial u_{h} / \partial t$ is the tangential derivative of $u_{h}$. Therefore, $\left|\left[\nabla u_{h}\right]_{\gamma}\right|=\left|\left[\partial u_{h} / \partial n\right]_{\gamma}\right|$ if $\gamma \in \mathcal{E}_{h, 0}$. The estimates (5.4) and (5.5) together with the shape regularity of the partition $\mathcal{P}_{h}$ imply

$$
\left\|\nabla u_{h}-G_{h} u_{h}\right\|_{0 ; K}^{2} \leq C h_{K}^{2} \sum_{\gamma \in \mathcal{E}_{\overparen{K}}}\left[\frac{\partial u_{h}}{\partial n}\right]_{\gamma}^{2} \leq C \sum_{\gamma \in \mathcal{E}_{\widetilde{K}}} h_{\gamma} \int_{\gamma}\left[\frac{\partial u_{h}}{\partial n}\right]_{\gamma}^{2} d s .
$$

Let $K \in \mathcal{P}_{h}$ be such that $\mathcal{E}(K) \cap \mathcal{E}_{h, C}=\emptyset$. It follows from (5.6) and definitions of $\eta_{K}$ and $R_{\gamma}$ that

$$
\eta_{K}^{2} \leq C \sum_{\gamma \in \mathcal{E}_{\widetilde{K}}} h_{\gamma}\left\|R_{\gamma}\right\|_{0 ; \gamma}^{2}
$$

Consider now the case when the element $K$ has at least one side lying on the boundary $\Gamma_{C}$. Let $\gamma \in \mathcal{E}(K) \cap \mathcal{E}_{h, C}$. Apply the triangle inequality to get

$$
\begin{aligned}
\eta_{K, G}^{2} & =\left\|\nabla u_{h}-G_{h} u_{h}\right\|_{0 ; K}^{2}+\sum_{\gamma \in \mathcal{E}(K) \cap \mathcal{E}_{h, C}} h_{\gamma}\left\|G_{h} u_{h} \cdot n_{\gamma}+g \lambda_{h}\right\|_{0 ; \gamma}^{2} \\
& \leq\left\|\nabla u_{h}-G_{h} u_{h}\right\|_{0 ; K}^{2}+\sum_{\gamma \in \mathcal{E}(K) \cap \mathcal{E}_{h, C}} h_{\gamma}\left(\left\|\left(\nabla u_{h}-G_{h} u_{h}\right) \cdot n_{\gamma}\right\|_{0 ; \gamma}+\left\|R_{\gamma}\right\|_{0 ; \gamma}\right)^{2} \\
& \leq\left\|\nabla u_{h}-G_{h} u_{h}\right\|_{0 ; K}^{2}+\sum_{\gamma \in \mathcal{E}(K) \cap \mathcal{E}_{h, C}} 2 h_{\gamma}\left(\left\|\left(\nabla u_{h}-G_{h} u_{h}\right) \cdot n_{\gamma}\right\|_{0 ; \gamma}^{2}+\left\|R_{\gamma}\right\|_{0 ; \gamma}^{2}\right) .
\end{aligned}
$$

From an inverse inequality and (5.6) we have

$$
\begin{aligned}
h_{\gamma}\left\|\left(\nabla u_{h}-G_{h} u_{h}\right) \cdot n_{\gamma}\right\|_{0 ; \gamma}^{2} & \leq h_{K}\left\|\nabla u_{h}-G_{h} u_{h}\right\|_{0 ; \partial K}^{2} \\
& \leq C\left\|\nabla u_{h}-G_{h} u_{h}\right\|_{0 ; K}^{2} \\
& \leq C \sum_{\gamma^{\prime} \in \mathcal{E}_{\widetilde{K}}} h_{\gamma^{\prime}}\left\|R_{\gamma^{\prime}}\right\|_{0 ; \gamma^{\prime}}^{2} .
\end{aligned}
$$

Inserting (5.6) and (5.9) into (5.8) concludes the proof.

From Lemma 5.1 and the inequality (5.2) we obtain

$$
\begin{aligned}
\eta_{G}^{2} \leq & C\left(\left\|u-u_{h}\right\|_{1 ; \Omega}^{2}+\sum_{\gamma \in \mathcal{E}_{h, C}} h_{\gamma}\left\|\lambda-\lambda_{h}\right\|_{0 ; \gamma}^{2}\right. \\
& \left.+\sum_{K \in \mathcal{P}_{h}} h_{K}^{2}\left\|r_{K}-\overline{r_{K}}\right\|_{0 ; K}^{2}+\sum_{\gamma \in \mathcal{E}_{h, C}} h_{\gamma}\left\|R_{\gamma}-\overline{R_{\gamma}}\right\|_{0 ; \gamma}^{2}\right) .
\end{aligned}
$$

Since linear elements are used, the term $\sum_{K \in \mathcal{P}_{h}} h_{K}^{2}\left\|r_{K}-\overline{r_{K}}\right\|_{0 ; K}^{2}$ can be replaced by $\sum_{K \in \mathcal{P}_{h}} h_{K}^{2}\|f-\bar{f}\|_{0, K}^{2}$, and the term $\sum_{\gamma \in \mathcal{E}_{h, C}} h_{\gamma}\left\|R_{\gamma}-\overline{R_{\gamma}}\right\|_{0 ; \gamma}^{2}$ can be replaced 
by $\sum_{\gamma \in \mathcal{E}_{h, C}} h_{\gamma} g^{2}\left\|\lambda_{h}-\overline{\lambda_{h}}\right\|_{0, \gamma}^{2}$, for discontinuous piecewise polynomial approximations $\bar{f}$ and $\overline{\lambda_{h}}$. The term $\sum_{K \in \mathcal{P}_{h}} h_{K}^{2}\|f-\bar{f}\|_{0, K}^{2}$ is clearly of higher order than $O\left(h^{2}\right)$. Numerical evidence from the next section shows that $\sum_{\gamma \in \mathcal{E}_{h, C}} h_{\gamma}\left\|\lambda-\lambda_{h}\right\|_{0 ; \gamma}^{2}$, and $\sum_{\gamma \in \mathcal{E}_{h, C}} h_{\gamma} g^{2}\left\|\lambda_{h}-\overline{\lambda_{h}}\right\|_{0, \gamma}^{2}$ as well, can be expected to be of higher order than $\| u-$ $u_{h} \|_{1 ; \Omega}^{2}$.

\section{Numerical experiment}

In this section, we show some numerical results in solving the following problem: Find $u \in H^{1}(\Omega)$ such that

$$
\int_{\Omega}[\nabla u \cdot \nabla(v-u)+u(v-u)] d x+g \int_{\Gamma}|v| d s-g \int_{\Gamma}|u| d s \geq \int_{\Omega} f(v-u) d x \quad \forall v \in H^{1}(\Omega) .
$$

We choose $\Omega=[0,1] \times[0,1], \Gamma_{C}=\Gamma, g=1$ and $f=-\Delta w+w$ with

$$
w(\boldsymbol{x})=\arctan (20(r-1 / 2)), \quad r^{2}=\left(x_{1}-0.8\right)^{2}+\left(x_{2}+0.2\right)^{2} .
$$

Triangular partitioning and linear elements are used in the experiment. The implementation was performed in MATLAB and a seven-point Gauss-Legendre quadrature was used to compute the load vector on each triangle. Numerical integration over a general triangle is reduced that over a reference triangle through a linear transformation of the variables. On the reference triangle

$$
\hat{K}=\{(\xi, \eta) \mid \xi \geq 0, \eta \geq 0,1-\xi-\eta \geq 0\},
$$

the seven-point Gauss-Legendre quadrature formula is defined by

$$
\int_{\hat{K}} F(\xi, \eta) d \xi d \eta \approx \sum_{i=1}^{7} \omega_{i} F\left(\xi_{i}, \eta_{i}\right),
$$

where the nodes $\left(\xi_{i}, \eta_{i}\right)$ and weights $\omega_{i}$ are given in Table 6.1. The discretized solution is computed by solving the equivalent minimization problem using an over-relaxation method with a relative error tolerance, in the maximum norm, of $10^{-6}$ (see [13], [14]).

\begin{tabular}{|r|r|r|r|}
\hline$i$ & $\xi_{i}$ & $\eta_{i}$ & $\omega_{i}$ \\
\hline 1 & $1 / 3$ & $1 / 3$ & $9 / 80$ \\
\hline 2 & $(6+\sqrt{15}) / 21$ & $(6+\sqrt{15}) / 21$ & $(155+\sqrt{15}) / 2400$ \\
\hline 3 & $(9-2 \sqrt{15}) / 21$ & $(6+\sqrt{15}) / 21$ & $(155+\sqrt{15}) / 2400$ \\
\hline 4 & $(6+\sqrt{15}) / 21$ & $(9-2 \sqrt{15}) / 21$ & $(155+\sqrt{15}) / 2400$ \\
\hline 5 & $(6-\sqrt{15}) / 21$ & $(6-\sqrt{15}) / 21$ & $(155-\sqrt{15}) / 2400$ \\
\hline 6 & $(9+2 \sqrt{15}) / 21$ & $(6-\sqrt{15}) / 21$ & $(155-\sqrt{15}) / 2400$ \\
\hline 7 & $(6-\sqrt{15}) / 21$ & $(9+2 \sqrt{15}) / 21$ & $(155-\sqrt{15}) / 2400$ \\
\hline
\end{tabular}

TABLE 6.1. Nodes and weights of 7-point Gauss-Legendre quadrature formula over reference triangle.

In order to show the effectiveness of the adaptive procedure we compare numerical convergence orders of the approximate solutions. We compute these orders by considering families of uniform and adaptively refined partitions. Consider a sequence 
of finite element solutions $u_{h}^{u n}$ based on uniform partitions of the domain $\Omega$. Starting with an initial coarse partition $\mathcal{P}_{1}$, we construct a family of nested meshes by subdividing each element into four congruent elements. The solution from the most refined mesh will be taken as the "exact" solution $u$, that will be used to compute the errors of the approximate solutions obtained on the other meshes.

Adaptive finite element solutions are obtained by the following algorithm:

1. Start with the initial partition $\mathcal{P}_{h}$ and corresponding finite element subspace $V_{h}$.

2. Compute the finite element solution $u_{h}^{a d} \in V_{h}$.

3. For each element $K \in \mathcal{P}_{h}$ compute the error estimator $\eta_{K}$ (residual or gradient recovery type).

4. Let $\eta=\frac{1}{N} \sum_{K \in \mathcal{P}_{h}} \eta_{K}$ with $N$ being the number of elements in partition $\mathcal{P}_{h}$. An element $K$ is marked for refinement if $\eta_{K}>\mu \eta$, where $\mu$ is a prescribed threshold. We take $\mu=0.5$.

5. Perform refinement and obtain a new triangulation $\mathcal{P}_{h}$.

6. Return to step 2 .

In the computation of the error indicator $\eta_{K}$ we make use of the multiplier $\lambda_{h}$ defined on $\Gamma$. In what follows we describe how $\lambda_{h}$ can be (approximately) recovered from the solution $u_{h}$ using characterization

$$
a\left(u_{h}, v_{h}\right)+\int_{\Gamma} g \lambda_{h} v_{h} d s=l\left(v_{h}\right) \quad \forall v_{h} \in V_{h}
$$

Denote by $\left\{\boldsymbol{x}^{i}\right\}_{i=1}^{m}$ the nodes of the partition $\mathcal{P}_{h}$ belonging to $\Gamma$, and let $\left\{\varphi_{i}\right\}_{i=1}^{m}$ be the basis functions corresponding to the nodes $\left\{\boldsymbol{x}^{i}\right\}$. We first determine a piecewise linear function

$$
\lambda_{h, 0}=\sum_{i=1}^{m} \lambda_{h, 0}^{i} \varphi_{i}
$$

by requiring an analogue of (6.1):

$$
a\left(u_{h}, v_{h}\right)+\int_{\Gamma} g \lambda_{h, 0} v_{h} d s=l\left(v_{h}\right) \quad \forall v_{h} \in V_{h} .
$$

Denote $\boldsymbol{\lambda}_{h, 0}=\left(\lambda_{h, 0}^{1}, \ldots, \lambda_{h, 0}^{m}\right)^{T}$. We then project the components of $\boldsymbol{\lambda}_{h, 0}$ onto the interval $[-1,1]$ to get $\boldsymbol{\lambda}_{h, 1}=\left(\lambda_{h, 1}^{1}, \ldots, \lambda_{h, 1}^{m}\right)^{T}$ :

$$
\lambda_{h, 1}^{i}= \begin{cases}-1 & \text { if } \lambda_{h, 0}^{i}<-1 \\ \lambda_{h, 0}^{i} & \text { if }-1 \leq \lambda_{h, 0}^{i} \leq 1 \\ 1 & \text { if } \lambda_{h, 0}^{i}>-1\end{cases}
$$

for $i=1,2, \ldots, m$. The piecewise linear approximation of the multiplier $\lambda_{h}$ on $\Gamma$ can be computed as

$$
\lambda_{h, 1}=\sum_{i=1}^{m} \lambda_{h, 1}^{i} \varphi_{i}
$$

We briefly comment on the method for finding $\boldsymbol{\lambda}_{h, 0}$. Let $n=\operatorname{dim} V_{h}$. Denote by $\boldsymbol{K}$ the standard $(n \times n)$ stiffness matrix and by $\boldsymbol{l} \in \mathbb{R}^{n}$ the standard load vector. Let 


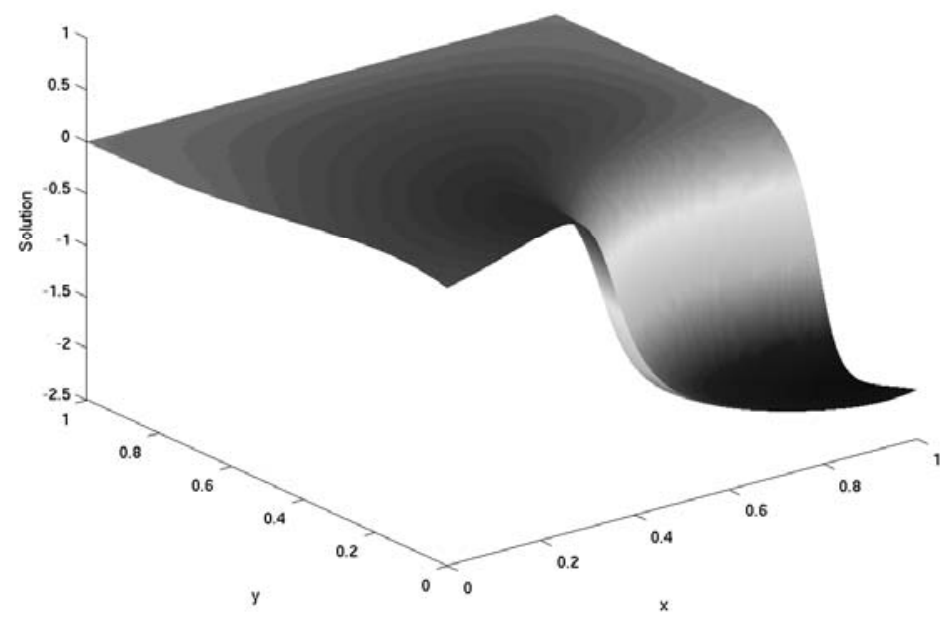

FIG. 6.1. Solution on a uniform mesh with 66,049 nodes.

$\boldsymbol{u} \in \mathbb{R}^{n}$ be the nodal value vector of the finite element solution $u_{h}$. Then the algebraic representation of (6.2) becomes

$$
(\boldsymbol{K u}, \boldsymbol{v})_{\mathbb{R}^{n}}+\left(\boldsymbol{M} \boldsymbol{\lambda}_{h, 0}, \boldsymbol{v}_{c}\right)_{\mathbb{R}^{m}}=(\boldsymbol{l}, \boldsymbol{v})_{\mathbb{R}^{n}} \quad \forall \boldsymbol{v} \in \mathbb{R}^{n},
$$

where $\boldsymbol{v}_{c}$ denotes the subvector of $\boldsymbol{v}$, containing the nodal values of $v_{h}$ at the nodes $\left\{\boldsymbol{x}^{i}\right\}_{i=1}^{m} \subset \Gamma$ and $\boldsymbol{M}$ is a tridiagonal $(m \times m)$ matrix. We can write $\boldsymbol{v}=\left(\boldsymbol{v}_{i}^{T}, \boldsymbol{v}_{c}^{T}\right)^{T} \in$ $\mathbb{R}^{n-m} \times \mathbb{R}^{m}$ by assuming that the components of $\boldsymbol{v}_{c}$ are listed last. We similarly split $\boldsymbol{l}$ to $\boldsymbol{l}_{i}$ and $\boldsymbol{l}_{c}$. This decomposition yields a block structure for $\boldsymbol{K}$,

$$
\boldsymbol{K}=\left(\begin{array}{ll}
\boldsymbol{K}_{i i} & \boldsymbol{K}_{i c} \\
\boldsymbol{K}_{c i} & \boldsymbol{K}_{c c}
\end{array}\right) .
$$

Then (6.4) is equivalent to the following two relations:

$$
\begin{aligned}
\boldsymbol{K}_{i i} \boldsymbol{u}_{i}+\boldsymbol{K}_{i c} \boldsymbol{u}_{c} & =\boldsymbol{l}_{i}, \\
\boldsymbol{K}_{c i} \boldsymbol{u}_{i}+\boldsymbol{K}_{c c} \boldsymbol{u}_{c}+\boldsymbol{M} \boldsymbol{\lambda}_{h, 0} & =\boldsymbol{l}_{c} .
\end{aligned}
$$

Once the approximate solution $u_{h}$ is computed, we can obtain from the second relation that

$$
\boldsymbol{\lambda}_{h, 0}=\boldsymbol{M}^{-1}\left(\boldsymbol{l}_{c}-\boldsymbol{K}_{c i} \boldsymbol{u}_{i}-\boldsymbol{K}_{c c} \boldsymbol{u}_{c}\right) .
$$

We start with a coarse triangulation shown on the left plot in Figure 6.2. Here, the interval $[0,1]$ is divided into $1 / h$ equal parts with $h=1 / 4$ which is successively halved. The numerical solution corresponding to $h=1 / 256$ is taken as the "exact" solution $u$. The "exact" solution is shown in Figure 6.1.

We use the regular refinement technique (red-blue-green refinement), where the triangle is divided into four triangles by joining the midpoints of edges and adjacent 
triangles are refined in order to avoid hanging nodes. For a detailed description of this and other refinement techniques currently used see e.g. [15] and references therein. Also, in order to improve the quality of triangulation, a smoothing procedure is used after each refinement. For each triangle $K$ of the triangulation we compute the triangle quality measure defined by

$$
Q(K)=\frac{4 \sqrt{3} \operatorname{area}(K)}{h_{1}^{2}+h_{2}^{2}+h_{3}^{2}}
$$

where $h_{i}, i=1,2,3$, are the side lengths of the triangle $K$. Note that $Q(K)=1$ if $h_{1}=h_{2}=h_{3}$. A triangle is viewed to be of acceptable quality if $Q>0.6$, otherwise we modify the mesh by moving the interior nodes toward the center of mass of the polygon formed by the adjacent triangles.

We use $u_{h}^{u n}$ for finite element solutions on uniform meshes, and $u_{h}^{a d}$ for finite element solutions on adaptive meshes. Since adaptive solutions are involved, numerical solution errors will be plotted against the number of degrees of freedom, rather than the meshsize.

We use an adaptive procedure based on both residual type and gradient recovery type estimates to obtain a sequence of approximate solutions $u_{h}^{a d}$. The adaptive finite element mesh after 5 adaptive iterations is shown on the right plot in Figure 6.2. Figures 6.3 and 6.4 contain the error values $\left\|u-u_{h}^{u n}\right\|_{1 ; \Omega}$ and $\left\|u-u_{h}^{a d}\right\|_{1 ; \Omega}$. We observe a substantial improvement of the efficiency using adaptively refined meshes. The errors $\left\|u-u_{h}^{a d}\right\|_{1 ; \Omega}$ computed using residual type and gradient recovery type estimators are shown in Figure 6.5. Table 6.2 contains the values of $C_{I}$ computed for uniform and adaptive solutions:

$$
C_{I}=\frac{\left\|u-u_{h}\right\|_{1 ; \Omega}}{\eta_{I}}, \eta_{I}=\left(\sum_{K} \eta_{K, I}^{2}\right)^{1 / 2}, \quad I \in\{R, G\} .
$$

It is clear from Table 6.2 that for this numerical example, the gradient recovery type error estimator provides a better prediction of the true error than the residual type error estimator, a phenomenon observed in numerous references. Note that implementation of the gradient recovery type error estimator is simpler than that of the residual type error estimator.

To have an idea of the convergence behavior of the discrete Lagrange multipliers, we analyze the errors $\left\|\lambda-\lambda_{h}\right\|_{0 ; \Gamma}$ corresponding to the sequence of uniform refinements. Here, $\lambda$ is the Lagrange multiplier corresponding to the parameter $h=1 / 256$. Figure 6.6 provides the error values $\left\|u-u_{h}^{u n}\right\|_{1 ; \Omega}$ and $h^{1 / 2}\left\|\lambda-\lambda_{h}\right\|_{0 ; \Gamma}$. The numerical convergence order of $h^{1 / 2}\left\|\lambda-\lambda_{h}\right\|_{0 ; \Gamma}$ is obviously higher than that of $\left\|u-u_{h}^{u n}\right\|_{1 ; \Omega}$, indicating that the second term in the efficiency bound (5.10) is expected to be of higher order compared to the first term $\left\|u-u_{h}\right\|_{1 ; \Omega}^{2}$. Graphs of $\lambda_{h, 0}$ and $\lambda_{h, 1}$ with $h=1 / 256$ are provided in Figures 6.7 and 6.8.

Acknowledgment. We thank the two referees for their comments on the first version of the paper.

\section{REFERENCES}

[1] M. Ainsworth and J.T. Oden, A Posteriori Error Estimation in Finite Element Analysis, John Wiley \& Sons, Inc., New York, 2000. 

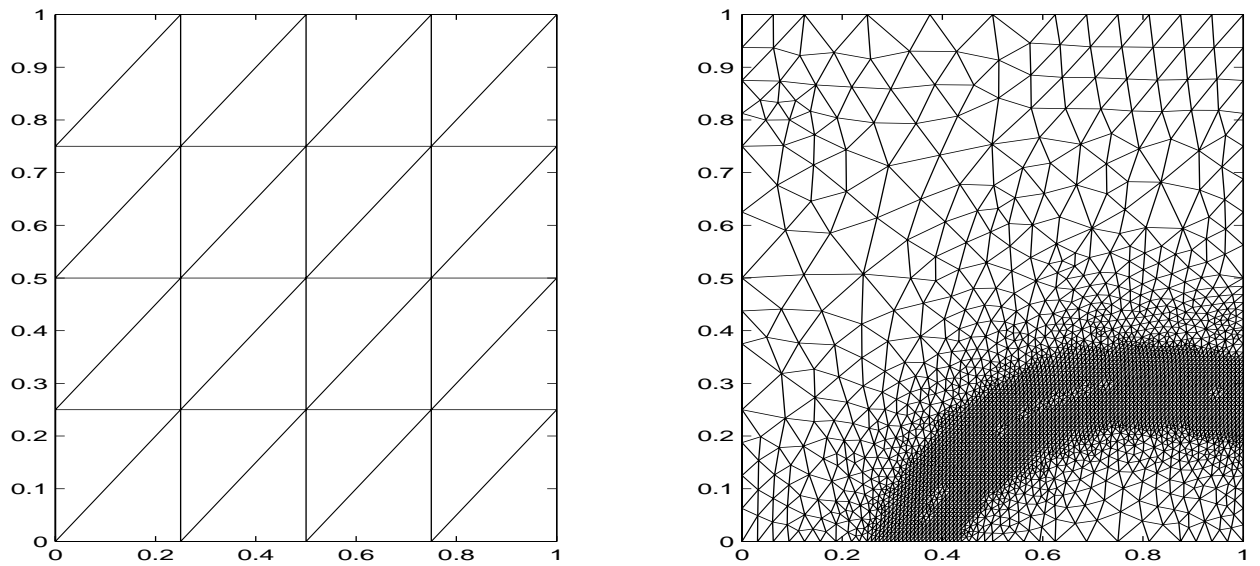

FIG. 6.2. Initial partition and locally refined partition after 5 iterations.

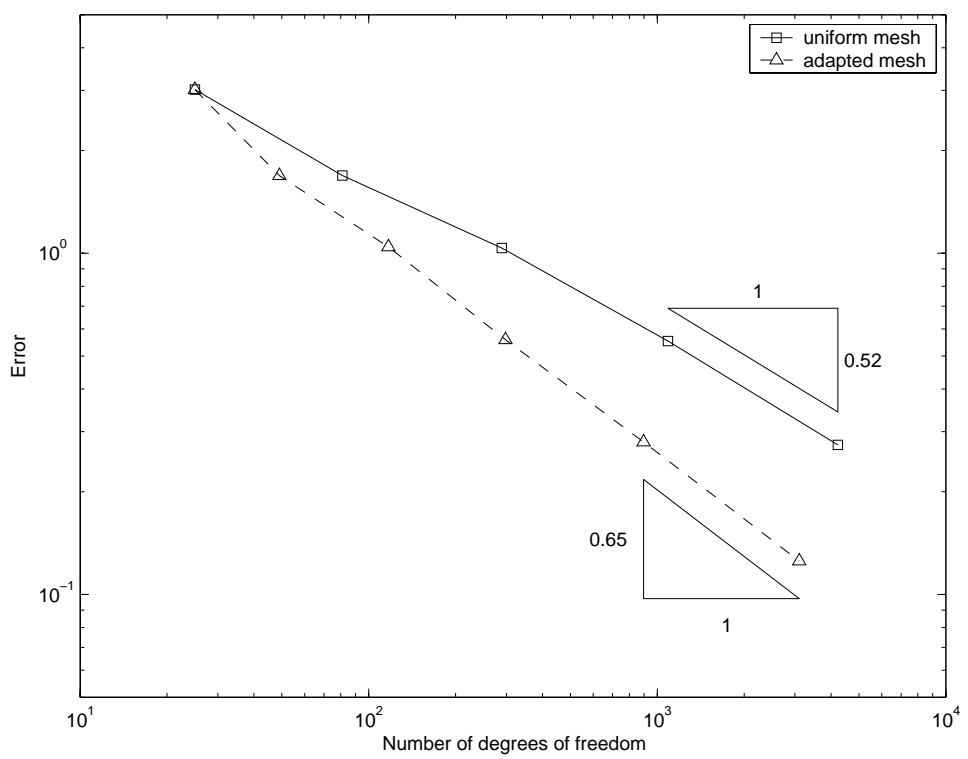

FIG. 6.3. Residual type estimator. $\left\|u-u_{h}^{u n}\right\|_{1 ; \Omega}(\square)$ vs. $\left\|u-u_{h}^{a d}\right\|_{1 ; \Omega}(\triangle)$.

\begin{tabular}{|l|r|r|r|r|r|r|}
\hline$h$ & $1 / 4$ & $1 / 8$ & $1 / 16$ & $1 / 32$ & $1 / 64$ & $1 / 128$ \\
\hline$C_{R}^{u n}$ & $1.13 \mathrm{e}-01$ & $1.23 \mathrm{e}-01$ & $1.44 \mathrm{e}-01$ & $1.47 \mathrm{e}-01$ & $1.44 \mathrm{e}-01$ & $1.29 \mathrm{e}-01$ \\
\hline$C_{R}^{a d}$ & $1.13 \mathrm{e}-01$ & $1.23 \mathrm{e}-01$ & $1.45 \mathrm{e}-01$ & $1.46 \mathrm{e}-01$ & $1.39 \mathrm{e}-01$ & $1.21 \mathrm{e}-01$ \\
\hline$C_{G}^{u n}$ & $1.17 \mathrm{e}+00$ & $8.23 \mathrm{e}-01$ & $8.56 \mathrm{e}-01$ & $8.89 \mathrm{e}-01$ & $9.18 \mathrm{e}-01$ & $8.48 \mathrm{e}-01$ \\
\hline$C_{G}^{a d}$ & $1.17 \mathrm{e}+00$ & $8.17 \mathrm{e}-01$ & $8.52 \mathrm{e}-01$ & $8.76 \mathrm{e}-01$ & $8.80 \mathrm{e}-01$ & $7.97 \mathrm{e}-01$ \\
\hline
\end{tabular}

TABle 6.2. Constants $C_{R}$ and $C_{G}$. 


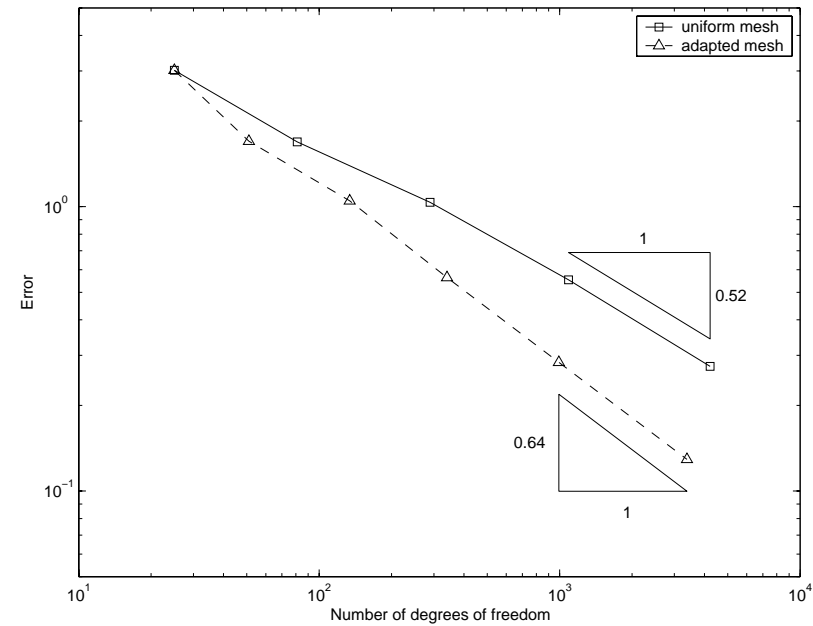

FIG. 6.4. Gradient recovery type estimator. $\left\|u-u_{h}^{u n}\right\|_{1 ; \Omega}(\square)$ vs. $\left\|u-u_{h}^{a d}\right\|_{1 ; \Omega}(\triangle)$.

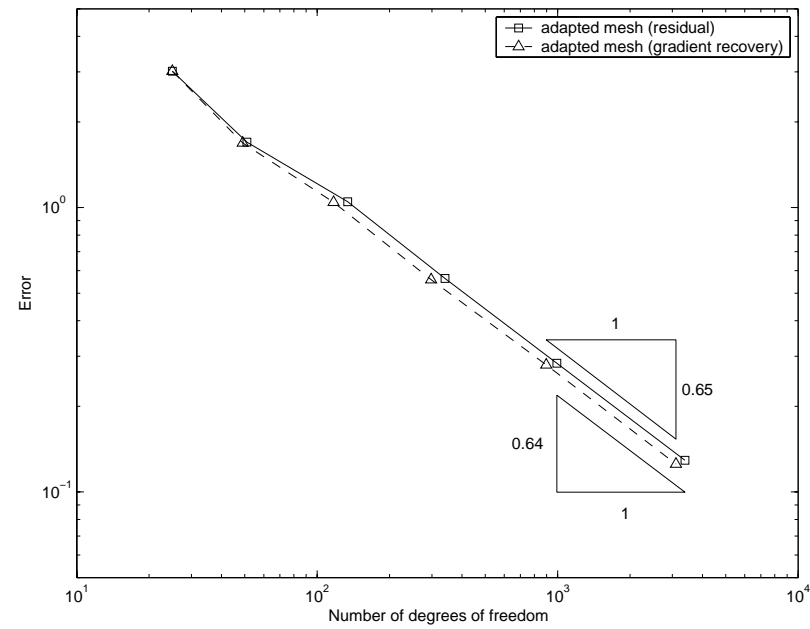

FIG. 6.5. $\left\|u-u_{h}^{a d}\right\|_{1 ; \Omega}$ for residual type estimator ( $\square$ ) vs. $\left\|u-u_{h}^{a d}\right\|_{1 ; \Omega}$ for gradient recovery type estimator $(\triangle)$.

[2] S. Bartels and C. Carstensen, Each averaging technique yields reliable a posteriori error control in FEM on unstructured grids. Part II: Higher order FEM, Math. Comp., 71:971-994, 2002.

[3] S. Bartels and C. Carstensen, Averaging techniques yield reliable a posteriori finite element error control for obstacle problems, preprint.

[4] C. Bernardi and V. Girault, A local regularization operator for triangular and quadrilateral finite elements, SIAM J. Numer. Anal., 35:1893-1916, 1998.

[5] V. Bostan, W. Han, and B.D. Reddy, A posteriori error analysis for elliptic variational inequalities of the second kind, in Computational Fluid and Solid Mechanics 2003, Proceedings of Second MIT Conference on Computational Fluid and Solid Mechanics, June 17-20, ed., K.J. Bathe, 1867-1870. 2003.

[6] V. Bostan, W. Han and B.D. Reddy, A posteriori error estimation and adaptive solution of elliptic variational inequalities of the second kind, submitted. 


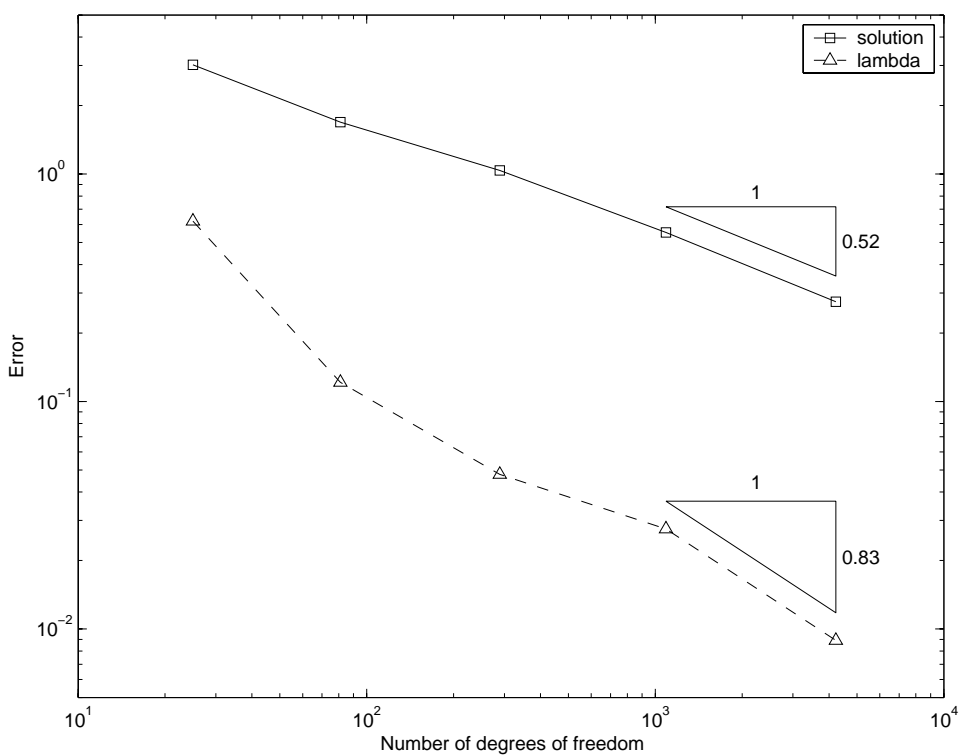

FIG. 6.6. $\left\|u-u_{h}^{u n}\right\|_{1 ; \Omega}(\square)$ vs. $h^{1 / 2}\left\|\lambda-\lambda_{h}\right\|_{0 ; \Gamma}(\triangle)$.
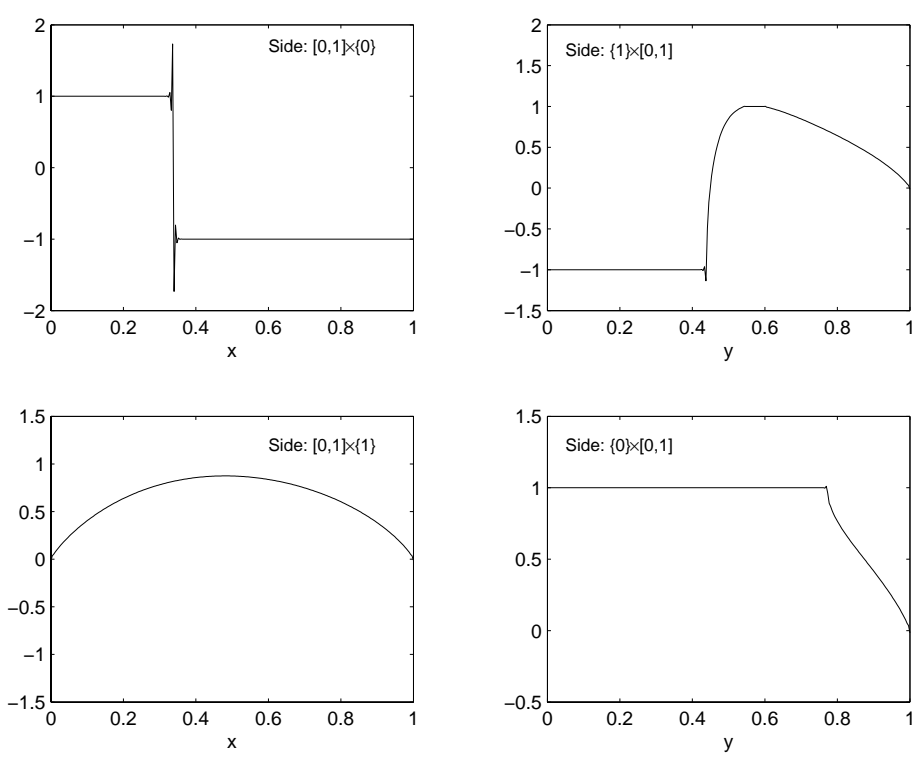

FIG. 6.7. Plots of $\lambda_{h, 0}$.

[7] C. Carstensen, Quasi-interpolation and a posteriori analysis in finite element methods, RAIRO Math. Model. Num., 33:1187-1202, 1999.

[8] C. Carstensen and S. Bartels, Each averaging technique yields reliable a posteriori error control in FEM on unstructured grids. Part I: Low order conforming, nonconforming, and mixed FEM, Math. Comp., 71:945-969, 2002. 

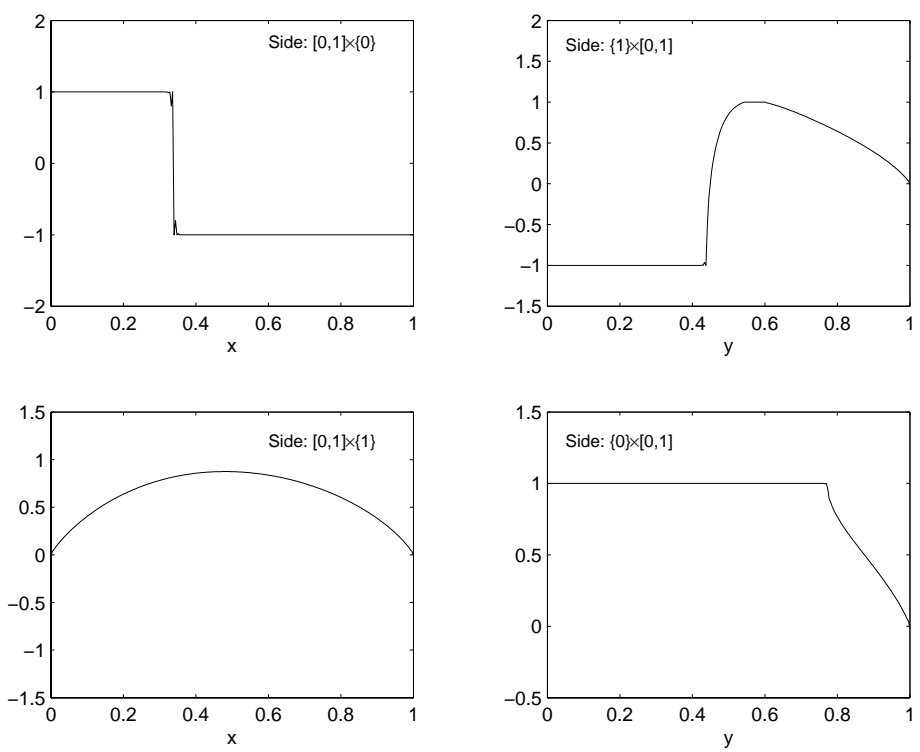

FIG. 6.8. Plots of $\lambda_{h, 1}$.

[9] C. Carstensen and R. Verfürth, Edge residual dominate a posteriori error estimates for low order finite element methods, SIAM J. Numer. Anal., 36:1571-1587, 1999.

[10] Z. Chen and R.H. Nochetto, Residual type a posteriori estimates for elliptic obstacle problems, Numer. Math., 84:527-548, 2000.

[11] Ph. Clément, Approximation by finite element functions using local regularization, RAIRO Numerical Analysis, R-2:77-84, 1975.

[12] I. Ekeland and R. Temam, Convex Analysis and Variational Problems, North-Holland, Amsterdam, 1976.

[13] R. Glowinski, Numerical Methods for Nonlinear Variational Problems, Springer-Verlag, NewYork, 1984.

[14] R. Glowinski, J.L. Lions, and R. Trémolières, Numerical Analysis of Variational Inequalities, North-Holland, Amsterdam, 1981.

[15] R. Verfürth, A Review of A Posteriori Error Estimation and Adaptive Mesh Refinement Techniques, Wiley and Teubner, 1996.

[16] N. Yan and A. Zhou, Gradient recovery type a posteriori error estimates for finite element approximations on irregular meshes, Comput. Meth. Appl. Mech. Engrg., 190:4289-4299, 2001.

[17] N. Yan, A posteriori error estimators of gradient recovery type for elliptic obstacle problems, Adv. Comput. Math., 15:333-362, 2001.

[18] Z. Zhang, A posteriori error estimates on irregular grids based on gradient recovery, Adv. Comput. Math., 15:363-374, 2001.

[19] O.C. Zienkiewicz and J.Z. Zhu, A simple error estimator and adaptive procedure for practical engineering analysis, Int. J. Numer. Meth. Engrg., 24:337-357, 1987.

[20] O.C. Zienkiewicz and J.Z. Zhu, The superconvergent patch recovery and a posteriori error estimates. Part 1: The recovery technique, Int. J. Numer. Meth. Engrg., 33:1331-1364, 1992.

[21] O.C. Zienkiewicz and J.Z. Zhu, The superconvergent patch recovery and a posteriori error estimates. Part 2: Error estimates and adaptivity, Int. J. Numer. Meth. Engrg., 33:1365$1382,1992$. 